\title{
Models for the Logical Topology Design Problem $^{\star}$
}

\author{
Nicolas Puech, Josué Kuri, and Maurice Gagnaire \\ École Nationale Supérieure des Télécommunications, \\ Computer Science and Networks Department, \\ 46, Rue Barrault, 75632 Paris cedex 13, France \\ \{npuech|kuri|gagnaire\} @enst.fr
}

\begin{abstract}
We address the logical topology design problem (LTD) in WDM transport networks under static traffic assumptions. We start with one of the standard MILP formulations of the LTD problem that aims at optimizing the network congestion. We propose an improvement to this model that additionally optimizes the average hop count. We then derive a new MILP model that compels the traffic to be atomically routed. This last model enables fair comparisons of solutions obtained with MILP formulations and with metaheuristic algorithms. The latter allow us to deal with large size networks whereas the former are limited by their computational complexity. In this paper Tabu Search is used to tackle the LTD problem. We compare and discuss the logical topologies computed by the various methods described in the paper.
\end{abstract}

\section{Introduction}

The network design problem consists of defining an optimal network configuration that fulfills specified traffic demands under technical and economical constraints [1]. Optimality is typically defined as the minimization of the network cost, maximization of specific network performance parameters (e.g. availability, call blocking, average packet delay, network congestion, etc.) or maximization of a function of the performance/cost ratio. In general, the minimum set of inputs for the design and dimensioning problem consists of a forecast of the traffic demands, topological data describing the physical links between nodes, and technical and cost information of the equipment and transmission infrastructure. The resulting optimal network configuration is typically described in terms of information on

* N. Puech is also with I.U.T., University Paris 5, 143 Avenue de Versailles, 75016 Paris, France, e-mail : puech@iut.univ-paris5.fr. This work has been funded by Alcatel R \& I, Marcoussis, France under Grant No. CI 578. Nicolas Puech has been funded by the French National Educational System (Éducation Nationale) and the University Paris 5 in the form of a sabbatical. Nicolas Puech would like to express his thanks to Mrs. Dominique Gascon (Director) and Mr. Serge Blumenthal (Statistics Department), both from the I.U.T., University Paris 5, France, for having decided in favor of his sabbatical. Josué Kuri has been partially supported by CONACyT Grant No. 122688.

E. Gregori et al. (Eds.): NETWORKING 2002, LNCS 2345, pp. 301-313 2002.

(C) Springer-Verlag Berlin Heidelberg 2002 
realization of traffic demands (e.g. working and protection paths of demands), location and configuration of equipment and network cost.

In Wavelength Division Multiplexing (WDM) transport networks, selective switching of wavelengths can be performed with current opto-electronic devices. Moreover, recent developments in optical devices make it possible to do this selective switching in the optical domain. From a network perspective, this functionality is of particular interest since it enables the development of networks that conveniently exploit the large transmission capacity of WDM systems. Clear optical channels, or lightpaths, that do not undergo opto-electronic conversion at intermediate nodes can be set between physically non-adjacent nodes in the network by assigning a wavelength to the lightpath and switching the wavelength optically. The information traveling on a lightpath is carried optically from end to end.

Because of equipment costs, fiber availability and switching capabilities at the network nodes, it is not possible to set up a full mesh of $N(N-1)$ lightpaths between the $N$ nodes of a network. Thus, a particular subset of lightpaths, out of the set of all possible lightpaths, must be selected. This subset is called the logical topology, or virtual topology, seen by the electronic switches at the network nodes. The traffic demands are realized on top of the logical topology by routing them through direct lightpaths, when they exist between the source and destination of the traffic, or through a concatenation of lightpaths otherwise. The logical topology is realized by routing the subset of lightpaths over the physical topology 1 and assigning wavelengths to these lightpaths.

Network design problems are in general very complex because the resulting optimization problems are numerically intractable even for networks with a small number of nodes. This inherent complexity frequently leads to solution approaches based on the decomposition of global design problems into subproblems of tractable complexity; each identified subproblem can be solved by a separate algorithm. Thus, a solution to the global problem is found by sequentially solving the subproblems, i.e. the solution to a first subproblem (output) is the input to the next one. This approach has been adopted, for example, in the design of SONET/SDH networks [1] and has also been used in the framework of WDM network planning [2].

Following a decomposition approach, three subproblems of the network design problem are usually considered in the literature:

- LTD (Logical Topology Design): the definition of the lightpaths to be set as the virtual topology and the routing of traffic demands over the virtual topology,

- LR (Lightpath Routing): the routing of lightpaths of the virtual topology over the physical topology,

- WA (Wavelength Assignment): the assignment of wavelengths to lightpaths. A drawback of the decomposition approach is that it precludes the enforcement of global optimality criteria because design choices leading to the optimality of one of the subproblems can be detrimental to the subsequent subproblems. It is possible to circumvent this drawback by first solving the subproblems sequen-

\footnotetext{
${ }^{1}$ or over a different server network layer.
} 
tially and then executing a second resolution; this second time, design information of the first phase can be exchanged as feedback between the algorithms that solve the subproblems so that "bad" design choices are known a priori and, consequently, are avoided. We adopted this approach in the resolution of the problems addressed in this paper. We first solve the LR problem considering a full-meshed logical topology for the considered networks. We then use the information about the routed lightpaths to prune the variable space associated with the MILP description of the LTD problem. This allows us to suppress a priori a number of solutions already considered unsuitable for the LTD problem. The size reduction resulting from this pruning process allows us to tackle networks up to 15 nodes whereas the MILP description considering all possible solutions only works for networks up to 7 nodes 2 . The pruning process is fully described and analyzed in [4].

In the following section we define the LTD problem and its tradeoffs. We then describe in Section 3 a multicommodity flow model that provides a mathematical description of the LTD problem as a Mixed Integer Linear Program (MILP). The aim is to minimize the network congestion, that is, to minimize the value of the traffic passing on the most congested lightpath. We show how the model may be improved in order to additionally minimize the average hop count. This model allows the traffic to be arbitrarily split over the chosen lightpaths. In Section 4 we derive a new model that enforces atomically routed traffic, that is, the traffic demand of one source-destination pair must follow the same route as a whole. The considered MILP models are intractable even for networks of small size which makes it often impossible to compute an exact solution to the LTD problem. When considering real size networks, one may be led to accept only approximate solutions to the problem, provided these are not too far from the exact solutions. Metaheuristic methods compute such approximate solutions for large size networks. As such, they are an interesting alternative to MILP based models. In Section 5, we describe a variant of the Tabu Search (TS) metaheuristic that we used for our study. TS is commonly used in optimization, particularly in the field of network planning 56. Like the model described in Section 4, TS enforces atomic routing of the traffic.

In Section [6] the solutions computed by these various algorithms are compared. We first evaluate the improvement brought by the hop count minimization. In order to evaluate the quality of the solutions computed by TS, it is interesting to compare them to the ones computed by the two preceding MILP models. The first model provides a lower bound on congestion, whether the routing is atomic or not. The second model provides a fair comparison since it takes into account atomic routing as is the case with TS. We will then compare the results computed by the three algorithms described previously. We will notice that TS reaches often the best possible solution. We hence legitimize the use of TS for large size networks.

\footnotetext{
${ }^{2}$ We used the OSL ILP solver [3] on a Sun Ultra 5 workstation with 128 Mbit RAM for our simulations. We limited our experiments to $10^{8}$ iterations of the solver. In most cases an optimal solution was found before reaching this limit.
} 


\section{Logical Topology Design}

The design of a logical topology and the routing of traffic demands over the topology can be formulated as an optimization problem. Optimality criteria are typically defined in terms of either network implementation cost or performance metrics [7]. Moreover, the constraints of the problem usually come from hardware limitations imposed by transmission and switching equipments.

Logical topology design in WDM transport networks has been extensively investigated in recent years $2,5,8910,11]$. Most of the proposed models aim at optimizing network performance metrics such as network congestion, throughput and average transit delay, rather than cost metrics. Focus on network performance criteria stems from the fact that the logical topology is the network layer directly seen by the electronic switches, which are typically expensive and, most importantly, offer limited capacity when compared to optical switches.

The logical topology design involves two tradeoffs: one between performance and network implementation costs and another one between capacity allocation in the electronic switches and the optical switches. Thus, a logical topology must be designed to meet performance and network cost criteria while satisfying hardware constraints at both the electronic and optical switching levels.

\section{Independently-Routed Multicommodity Flow Model}

Some multicommodity flow models have been developed to solve the LTD problems by mathematical means. Some of these models aim at solving the LTD, LR and WA problems simultaneously [11, while others consider either, LTD and LR [10] together, or LTD alone [8.9. Integrated models that jointly consider the three mentioned problems are expected to yield better solution 3 than models that address the problems separately. However, the complexity of integrated models precludes their use when solving problem instances of realistic size. The two multicommodity flow models presented in this paper consider the LTD problem alone. The LR and WA problems are not considered here though, as mentioned previously, we will prune the LTD variable space according to the feedback method described in [4].

We begin the presentation of the first multicommodity flow model by introducing the notation. We will keep the same notation for the second model in Section 4 and for the application of tabu search in Section 5

\section{Indices:}

$i, j \quad$ used as subscripts, denote the source and destination nodes of a lightpath.

$s, d \quad$ used as superscripts, denote the source and destination nodes of a traffic demand.

$\overline{{ }^{3} \text { with respect }}$ to specific global network performance and resource utilization criteria. 


\section{Problem parameters:}

$N$ the number of nodes in the network (the nodes are numbered $1,2, \ldots, N)$.

$\left(\lambda^{s d}\right)$ an $\left(\mathbb{R}_{+}\right)^{N \times N}$ matrix describing the amount of traffic flowing from each source node $s$ to each destination node $d$, expressed in some convenient units (e.g. Mbit/s). The matrix is not necessarily symmetrical.

$\delta_{\text {out }}$ the logical out-degree of the nodes in the network, i.e. the maximum number of lightpaths that can initiate at the electronic switch of the network nodes.

$\delta_{\text {in }}$ the logical in-degree of the nodes in the network, i.e. the maximum number of lightpaths that can be terminated at the electronic switch of the network nodes. It is often assumed that $\delta_{\text {out }}=\delta_{\text {in }}=\delta$, although this is not a strict requirement.

Variables:

$b_{i j} \quad$ a binary variable that indicates whether or not there is a lightpath from source node $i$ to destination node $j$ (1 and 0 respectively). Note that lightpaths are directed, therefore, $b_{i j}=1$ does not imply $b_{j i}=1$.

$\lambda_{i j}^{s d} \quad$ a real variable indicating the amount of traffic from source $s$ to destination $d$ passing through the lightpath going from $i$ to $j$.

Computed values:

$\lambda_{i j} \quad$ the total amount of traffic passing through the lightpath going from node $i$ to node $j$, i.e. $\lambda_{i j}=\sum_{s} \sum_{d} \lambda_{i j}^{s d} \forall i, j$.

$\lambda_{\max }$ the amount of traffic passing through the most congested lightpath, i.e. $\lambda_{\text {max }}=\max _{1 \leq i, j \leq N} \lambda_{i j}$. This term is referred to as the maximum congestion level in the network or simply, the congestion.

\section{Model MILP1}

This multicommodity flow model is partially based on the formulation presented in [8]. The model is defined in terms of $b_{i j}$ (binary) and $\lambda_{i j}^{s d}$ (real) variables, hence it is a MILP (Mixed Integer Linear Program). Once the problem is solved and the values of the variables are known, the variables $b_{i j}$ set to 1 indicate the lightpaths to be established and, for each traffic demand $\lambda^{s d}$, the values of the variables $\lambda_{i j}^{s d}$ indicate the amount of $s d$ traffic flowing on lightpath $i j$. The model is:

$$
\text { Minimize: } \lambda_{\max }
$$

\section{Subject to:}

Flow conservation at each node:

$$
\sum_{i} \lambda_{i j}^{s d}-\sum_{k} \lambda_{j k}^{s d}=\left\{\begin{array}{ll}
-\lambda^{s d}, & \text { if } j=s \\
\lambda^{s d}, & \text { if } j=d \\
0, & \text { otherwise }
\end{array} \quad \forall j, s, d\right.
$$


Total flow on a lightpath:

$$
\begin{aligned}
& \lambda_{i j}=\sum_{s} \sum_{d} \lambda_{i j}^{s d} \leq \lambda_{\max }, \forall i, j \\
& \lambda_{i j}^{s d} \leq b_{i j} \lambda^{s d}, \quad \forall i, j, s, d
\end{aligned}
$$

Degree constraints:

$$
\begin{gathered}
\sum_{j} b_{i j} \leq \delta_{\text {out }}, \forall i \\
\sum_{i} b_{i j} \leq \delta_{\text {in }}, \forall j
\end{gathered}
$$

Value range constraints:

$$
\begin{aligned}
\lambda_{i j}^{s d} \geq 0, \quad \forall i, j, s, d \\
b_{i j} \in\{0,1\}, \forall i, j
\end{aligned}
$$

The objective function (1) states that the model aims at minimizing $\lambda_{\max }$, the maximum congestion level in the network. Equation (2) is the flow conservation constraint which states that, for a traffic demand $\lambda^{s d}$, at every intermediate node $j(j \neq s, j \neq d)$, the amount of flow entering the node must be equal to the amount leaving it. At the source node $(j=s)$, the traffic only leaves the node, whereas at the destination node $(j=d)$, the traffic only enters the node. Equation (3) ensures that the total amount of traffic passing through any lightpath is at most equal to $\lambda_{\max }$. Equation (4) states that traffic can flow on a lightpath only if the lightpath exists. Moreover, for a traffic demand from $s$ to $d$, the amount of traffic flowing on the lightpath from $i$ to $j$ cannot be more than the total amount of the traffic demand. Equations (5) and (6) enforce the maximum logical out-degree and in-degree of the nodes, respectively. Note that some nodes with degree smaller that $\delta$ can be accepted in the solution. As we shall see later, this allows us to find virtual topologies that minimize the congestion while using as few lightpaths as possible. Finally, equations (7) and (8) constrain the flow indicator variables to be non-negative reals and the lightpath indicator variables $b_{i j}$ to be binary.

\section{Model MILP1b}

It may happen that multiple solutions minimize the congestion for the same problem instance. Thus, once the minimum possible congestion value $\lambda_{\max }^{*}$ has been found, one can look within the set of solutions for a solution that optimizes a different criterion. For example, a solution that minimizes the average hop count (the average number of lightpaths that the traffic demands traverse to go from source to destination) for a given $\lambda_{\max }^{*}$ can be found by modifying the preceding formulation in the following manner: 
- first, replace the objective function (1) by

$$
\text { Minimize: } \sum_{i} \sum_{j} \sum_{s} \sum_{d} \lambda_{i j}^{s d}
$$

- then, replace the variable $\lambda_{\max }$ in equation (3) by the value $\lambda_{\max }^{*}$.

This leads to a two-step optimization process. The improvement of this model with respect to the basic one is assessed in Section [6] where a comparison between the results obtained in step 1 and the ones computed in step 2 is provided.

\section{Atomically-Routed Multicommodity Flow Model}

The previous model allows a traffic demand $\lambda^{s d}$ to be arbitrarily split across multiple possible routes. While this property is useful to determine the lower bound of the congestion in the network, the routing of traffic demands obtained in this manner may have little practical relevance. Though existing packet-switched networks (e.g. ATM, Internet) provide a fine-grained switching granularity, this granularity cannot be used to arbitrarily split a traffic demand because of restrictions imposed by network protocols (e.g. avoiding packet reordering in TCP connections) or architectures.

We propose a model that allow the traffic demands to be atomically routed, i.e. the whole traffic from source $s$ to destination $d$ follows the same route through the network, as suggested in [10]. Metaheuristic methods usually assume atomic traffic routing, and it is fair to compare their solutions with the ones computed with this model rather than with the ones that allow traffic splitting like the model MILP1.

\section{Model MILP2}

The model is directly inferred from the model MILP1 with the introduction of new variables $\varphi_{i j}^{s d}=\lambda_{i j}^{s d} / \lambda^{s d}$ to describe the flow and an additional constraint to avoid traffic splitting: $\varphi_{i j}^{s d} \in\{0,1\}, \forall i, j, s, d$. Thus, the model becomes:

$$
\text { Minimize: } \lambda_{\max }
$$

\section{Subject to:}

constraints (2), (3), (4), (5), (6) and (8).

\section{Model MILP2b}

As in model MILP1, once the congestion value $\lambda_{\max }^{*}$ has been found, the objective function (10) can be replaced by 


\section{Minimize: $\sum_{i} \sum_{j} \sum_{s} \sum_{d} \varphi_{i j}^{s d}$}

in order to find a routing of demands that additionally minimizes the average hop count.

\section{Tabu Search: A Metaheuristic Approach}

The major drawback of multicommodity flow models is that they are computationally intractable even for small problem instances. Optimization algorithms for the LTD problem based on metaheuristics have recently gained interest because they are able to cope with problem instances of large size. Among others, simulated annealing [6], and Tabu Search (TS) [5] have been proposed to tackle the LTD problem. The computed solution however is not guaranteed to be optimal. These algorithms have the same aim as the multicommodity flow model presented in Section 4 (model MILP2) in the sense that they try to find a virtual topology with minimal congestion under given logical degree constraints and using atomic routing of traffic demands.

Tabu search has proven to be an effective metaheuristic method for LTD 56 . This section describes an optimization algorithm for the LTD problem based on TS that was initially proposed in [5]. A detailed description of the TS algorithm can be found in 12 .

We will summarize TS as follows. TS consists of exploring the space of solutions until a number of iterations is reached or until a specific cost criterion is satisfied. The exploration starts with an initial solution computed by another algorithm (among others, initial solution randomly choosen). At each iteration, TS computes a set (called neighborhood) of solutions derived from the current solution via perturbations applied to this solution. All the solutions of the neighborhood are evaluated (see below) and the best one is selected as the new current solution. In order to prevent the algorithm from cycling along the same series of current solutions, a tabu list is maintained. It contains a number of last visited solutions, which cannot be chosen as long as they belong to this list. This allows the algorithm to choose a solution worse than the current one, allowing it to escape from the local minima encountered during the search.

\section{Experimental Results}

An instance of the LTD problem is defined by a physical topology and a traffic matrix $\left(\lambda^{s d}\right)$. The physical topology corresponds to the nodes of the network and the fiber links connecting them.

The elements of the traffic matrix represent a forecast of the packet traffic demand between any two nodes in the network expressed in generic units. The solution to the problem is a set of lightpaths that constitute the virtual topology. 
An optimal solution is one that minimizes the traffic congestion in the virtual topology. For the experiments of this Section, we use a physical topology that corresponds to a hypothetical backbone network of 9 nodes.

Concerning the traffic demands, in a first step, we used two different traffic matrices. The first one, hereafter referred to as Matrix1, comes from a real traffic evaluation and has a particular element element which is much larger than the others and whose value is 847 traffic units. This value is the lowest bound that any atomic routing algorithm may reach for congestion. The average value of Matrix1 is 123.63 traffic units and the smallest value is 1 . In the second traffic matrix, that we call Matrix2, all the elements, except those of the diagonal, are $\lambda^{s d}=124.0 \forall s, d \quad s \neq d$. The purpose of this second matrix is to evaluate the results of the algorithms under the particular condition arising when all the nodes in the network exchange the same amount of traffic. Thus, we have two different problem instances: one represented by the physical topology and Matrix1, and one represented by the same topology and Matrix2.

Table 1. Congestion $\left(\lambda_{\max }\right)$ obtained with MILP1, MILP2 and TS.

\begin{tabular}{|c||r|r|c||r|r|c|}
\hline \multicolumn{1}{|c||}{} & \multicolumn{3}{|c||}{ Matrix 1 } & \multicolumn{3}{c|}{ Matrix 2 } \\
\hline Degree & MILP1 & MILP2 & TS & MILP1 & MILP2 & TS \\
\hline 3 & 752.16 & $(923.00)$ & 847.00 & 620.00 & 620.00 & 620.00 \\
4 & 524.00 & 847.00 & 847.00 & 372.00 & 496.00 & 496.00 \\
5 & 492.00 & 847.00 & 847.00 & 286.15 & 372.00 & 496.00 \\
6 & 492.00 & 847.00 & 847.00 & 233.58 & 248.00 & 372.00 \\
7 & 492.00 & 847.00 & 847.00 & 201.50 & 248.00 & 327.00 \\
\hline
\end{tabular}

Table 1 present:4 the congestion of the logical topologies obtained with MILP1, MILP2 and TS, respectively for Matrix1 and Matrix2 traffic demands, for degree values ranging from 3 to 7 . It is noticeable that TS always reaches the best possible value for Matrix1. Let us recall that TS, like MILP2, enforces atomic routing, that is, the whole traffic demand of a source-destination pair must follow the same route. Hence, in the case of atomic routing, the optimal congestion value is the greatest value of the traffic matrix. When the degree is 3 , TS even outperforms MILP2. Indeed, the value indicated in that case for MILP2 is the partial result obtained with the solver since the solver did not manage to find the optimal solution 847 before the maximum allowed number of iterations.

In the case of Matrix2, the difference in the congestion values computed by MILP1 and MILP2 is not so important as in the case of Matrix1. TS is quite good for degree 3 or 4 but reaches values higher than the ones found by MILP2. It seems that the case of a homogeneous traffic demand is more difficult to tackle.

It is also interesting to observe that allowing traffic to be independently routed allows us to reach far better congestion values in any case. This suggests that it might be interesting to study traffic splitting issues in future research.

Finally, in the case of Matrix1, it appears unnecessary to study logical topologies with degree higher than 3 since the lower bound for congestion is already found by the atomic routing algorithms, MILP2 or TS, when the degree is 3 . Conversely, in the Matrix2 case, the congestion is significantly reduced when the

\footnotetext{
${ }^{4}$ The value indicated for Matrix1, MILP2 and degree 3 was the one computed at the iteration limit (see footnote 3 ) and is not optimal.
} 
degree increases. However, increasing the logical degree increases the number of necessary lightpaths and, ultimately, the number of ports in the optical switches.

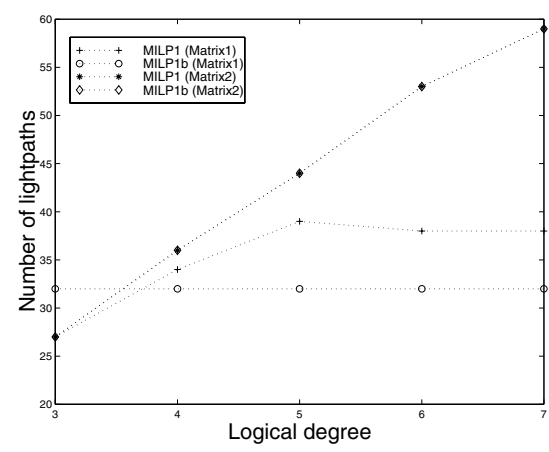

(a) MILP1 and MILP1b

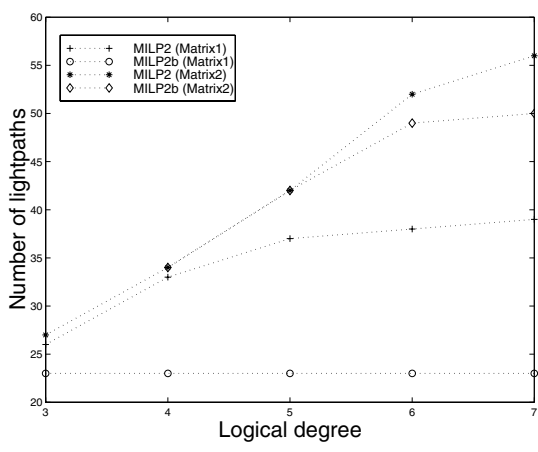

(b) MILP2 and MILP2b

Fig. 1. Required number of lightpaths when using MILP1, MILP1b, MILP2 and MILP2b models as a function of the logical degree (with Matrix 1 and with Matrix 2).

The results collected with Matrix1 seem to indicate that it has a very special traffic distribution hence leading to extreme results (among others, the maximal value of Matrix1 being far higher than the mean value, both algorithms reach this bound). That is why we had another series of experiments to compare the algorithms. We generated 10 traffic matrices randomly with uniformly distributed values in the range $0-1000$. We computed the congestion obtained for these matrices with MILP1, MILP2 and TS and observed the following results. Note that almost all computations lead with MILP2 had to be stopped due to iteration limit, hence the values obtained were not optimal. The average congestion (respectively standard deviation) obtained is 1726.2 (141.8) for MILP1, 2590.5 (396.0) for MILP2 and 1997.9 (152.9) for TS. The mean congestion increase from MILP1 to MILP2 is $50 \%$ and it is $16 \%$ from MILP1 to TS. This last result clearly shows the practical limit of MILP2 since TS allows us to get better values than the interrupted MILP2 search in much less time.

Figure 1 shows the required number of lightpaths computed by MILP1/ MILP1b and MILP2/MILP2b models as a function of the logical degree for both Matrix1 and Matrix2. The number of required lightpaths is smaller when using the two-step optimization MILP1b/MILP2b variants of the models. This result was expected since both MILP1b and MILP2b minimize the congestion and the average hop count. The difference between MILP1/MILP2 and their variants MILP1b/MILP2b is more important in the case of Matrix1, when the traffic demand is non homogeneous. Conversely, one can notice that in the case of a homogeneous traffic demand (Matrix2), the improvement of MILP1b/MILP2b 
is not very significant (it is null in the case of MILP1b) whereas there was a gain of up to $40 \%$ in the number of lightpaths in the case of Matrix1 with MILP2b.

Figure 2 shows the traffic distribution over the lightpaths computed by MILP1, MILP2, MILP2b and TS, for a logical topology with degree 4 and for Matrix1 and Matrix2. It is clear that MILP1 outperforms the other algorithms since it is allowed to split the traffic over several routes. The comparison can only be considered as fair between MILP2, MILP2b and TS, and clearly the curves corresponding to these methods are close and of the same shape. One can also notice on Figure 2 that MILP2b not only requires a smaller number of lightpaths to establish the traffic demand, but also computes a better lightpath usage : the 20 first lightpaths support more traffic than the corresponding ones computed by MILP2 or TS. MILP2b can be considered as more efficient in this respect.

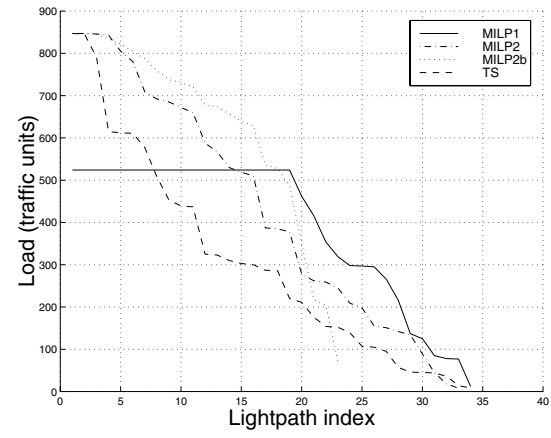

(a) Matrix 1

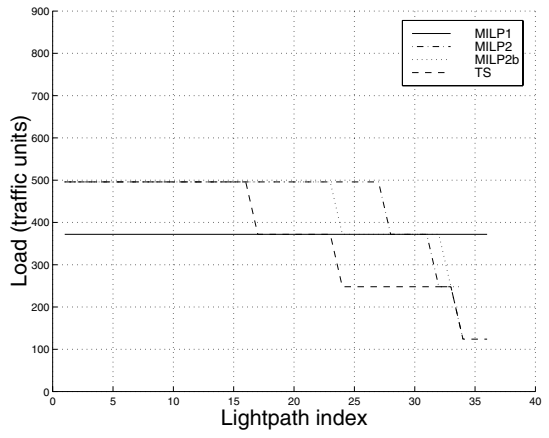

(b) Matrix 2

Fig. 2. Distribution of traffic load on the lightpaths of the virtual topology (degree=4) computed by MILP1 (which stands as a lower bound for congestion), MILP2, MILP2b and TS. Tests for Matrix1 and Matrix 2.

This series of experiments presents a fair comparison between a MILP model and TS and shows that the results obtained with TS are close to the ones computed with the other models. While one should not underestimate the difference that remains between TS and MILP2 (a 30\% congestion increase in the case of Matrix2, which is a kind of worst case), it is worth mentioning that in our experiments TS was 100 to 10000 times faster than the tested MILP models. Moreover, TS is able to compute solutions to the LTD problem for networks with as much as 150 nodes whereas MILP models become intractable for more than 15 nodes. The results obtained in this study plead in favor of TS which provides results fairly close to the optimal solution with far better computing times on the tested situations. It hence seems reasonable to compute approxi- 
mate solutions to the LTD problem with TS for real size networks. Moreover, it might be interesting to modify the TS algorithm so that it can additionally perform the average hop count minimization, since MILP1b and MILP2b show an improvement with respect to MILP1 and MILP2.

\section{Conclusion}

Starting from a standard multicommodity flow model describing the LTD problem, we have proposed a second optimization step in order to find solutions that additionally minimize the average hop count. Experiments have shown that this two-step approach leads to solutions requiring fewer lightpaths than the solutions found by the original model. We also derived a new model that enforces atomic traffic routing. This models serves as a base to compare solutions to the LTD problem obtained with MILP formulations and a TS metaheuristic. Experiments have shown that TS often reaches one of the best possible solutions. Hence TS, even though it is a method which does not ensure that one will find an optimal solution, may be a very good alternative to solve the LTD problem, especially since it enables one to deal with much larger problem instances than MILP based models which are computationally intractable. An improvement to the current TS LTD algorithm would consist of modifying the cost function in order to additionally minimize the average hop count, as done in the MILP models presented in the paper.

\section{References}

[1] Eurescom. Planning of Optical Networks. Deliverable 3 Project P709, Eurescom, 2000.

[2] E. Leonardi, M. Mellia, and M. Ajmone Marsan. Algorithms for the logical topology design in WDM all-optical networks. Optical Networks Magazine, 1(1):35-46, 2000.

[3] IBM Spatial and Optimization Solutions. IBM's Optimization Solutions and Library, version 3.

[4] J. Kuri, N. Puech, and M. Gagnaire. Resolution of a WDM optical network design problem using a decomposition approach and a size reduction method. ECUMN 2002, Colmar, France, 2002.

[5] M. Mellia, A. Nucci, A. Grosso, E. Leonardi, and M. Ajmone Marsan. Optimal design of logical topologies in wavelength-routed optical networks with multicast traffic. In Proceedings of INFOCOM 2001. IEEE Communications Society, 2001.

[6] R. Caberlon, W. Floris, and N. Puech. Logical topology design : a case study. Private communication, École Nationale Supérieure des télécommunications, 2001.

[7] M. Ammar, S. Cheung, C. Scoglio, I. Chlamtac, A. Faragó, and T. Zhang. Virtual path network design. In J. Roberts, U. Mocci, and J. Virtamo, editors, Broadband Network Teletraffic. Final report of action COST 242, pages 271-299. SpringerVerlag, Berlin Heidelberg, 1996.

[8] R. Ramaswami and K. N. Sivarajan. Design of logical topologies for wavelengthrouted optical networks. IEEE Journal on Selected Areas in Communications, 14(5):840-851, 1996. 
[9] B. Mukherjee, D. Banerjee, S. Ramamurthy, and A. Mukherjee. Some principles for designing a wide-area WDM optical network. IEEE/ACM Transactions on Networking, 4(5):684-696, 1996.

[10] D. Banerjee and B. Mukherjee. Wavelength-routed optical networks: Linear formulation, resource budgeting tradeoffs, and a reconfiguration study. IEEE/ACM Transactions on Networking, 8(5):598-607, 2000.

[11] R. Krishnaswamy and K. Sivarajan. Design of logical topologies: A linear formulation for wavelength-routed optical networks with no wavelength changers. IEEE/ACM Transactions on Networking, 9(2):186-198, 2001.

[12] F. Glover and M. Laguna. Tabu Search. Kluwer Academic Publishers, Boston, MA, 1997. 